\title{
Alterssicherungspolitik erfordert gute Renten und echte Reformen
}

\author{
JUDITH KERSCHBAUMER
}

Alterssicherungspolitik und Altersarmut haben sich zu einem zentralen sozialpolitischen Schwerpunktthema entwickelt, dem im Bundestagwahlkampf 2013 erhebliche Bedeutung zukommt. Dabei ist - neben der seit der Rentenreform des Jahres 2007 diskutierten „Rente mit 67“ - die Auseinandersetzung um das Sicherungsziel staatlicher Alterssicherungspolitik, also um das Rentenniveau, wieder in den Fokus gerückt. Eine wichtige Rolle spielen auch Forderungen nach einer Stärkung des sozialen Ausgleichs in der Rentenversicherung sowie nach einer Aufstockung der Erwerbsminderungsrenten. Mit der Rentenanpassung zum 1.7.2013 wurde das - 23 Jahre nach der Deutschen Einheit immer noch unterschiedliche - Rentenrecht in den alten und neuen Bundesländern wieder Gegenstand der politischen Debatte.

\section{Sinkendes Rentenniveau und steigende Altersarmut}

Mit der Rentenreform 2001, ${ }^{1}$ der sogenannten Riester-Renten-Reform, wurde von der ausgabenorientierten Einnahmepolitik und damit von der Maxime, dass das angestrebte Sicherungsziel den Beitragssatz bestimmt, Abschied genommen. Stattdessen wurde nunmehr die sogenannte Beitragssatzstabilität zur handlungsleitenden Norm erklärt ( $\$ 154$ Abs. 3 SGB VI). Dabei geht es um das Ziel, den Rentenversicherungsbeitrag bis zum Jahr 2020 auf maximal $20 \%$ und bis zum Jahr 2030 auf höchstens $22 \%$ des beitragspflichtigen Arbeitsentgelts zu begrenzen. Damit ging die Politik zu einer einnahmeorientierten Ausgabenpolitik über.

Das Rentenniveau stand lange Zeit nicht mehr im Mittelpunkt staatlicher Alterssicherungspolitik. Erst die sinkenden Rentenzahlbeträge ${ }^{2}$ haben ein Bewusstsein dafür geschaffen, dass Altersarmut - obwohl von der Bundesregierung zuerst als gesellschaftliches Problem negiert - doch für immer breitere Bevölkerungsschichten zu einer zunehmenden Bedrohung wird (vgl. dazu auch Seils in diesem Heft). Eine Gefahr für die Akzep-

TABELLE 1

\section{Rentenzahlbeträge der Altersrenten im Jahr 2011 nach Bestand und Zugang}

Angaben in Euro

\begin{tabular}{|c|c|c|c|c|}
\hline & \multicolumn{2}{|c|}{ Männer } & \multicolumn{2}{|c|}{ Frauen } \\
\hline & Alte Bundesländer & Neue Bundesländer & Alte Bundesländer & Neue Bundesländer \\
\hline Bestand & 987 & 1.058 & 495 & 711 \\
\hline Zugang & 868 & 867 & 487 & 681 \\
\hline
\end{tabular}

Quelle: Statistik der Deutschen Rentenversicherung, Deutsche Rentenversicherung Bund, DRV-Schriften Band 22, Rentenversicherung in Zeitreihen, Oktober 2012, S. 126f., $204 \mathrm{f}$.

tanz der Gesetzlichen Rentenversicherung (GRV) liegt in dieser Armutsdebatte selbst. Die GRV unterliegt dem Versicherungs- und Äquivalenzprinzip und gewährt grundgesetzlich in bestimmtem Umfang geschützte Ansprüche und Anwartschaften. Je mehr dem Sozialstaat jedoch die Rolle eines Armutsvermeidungssystems übertragen wird, in dem Fürsorgeleistungen von einer Bedürftigkeitsprüfung abhängen, desto geringer wiegen einklagbare Ansprüche und Anwartschaften und desto mehr wird die Ausgestaltung der GRV in das politische Belieben der jeweiligen politischen Mehrheitsverhältnisse gestellt - mit der absehbaren Folge, dass das Vertrauen der Bevölkerung in die GRV sukzessive ausgehöhlt wird.

Die Wirkung der Reformen der letzten zehn bis 20 Jahre lässt sich am Vergleich von Bestand und Zugang der Rentenzahlbeträge bei Männern besonders deutlich erkennen (Tabelle 1): Während bei Männern der durchschnittliche Zahlbetrag der Zugangsrenten des Jahres 2011 in den alten Bundesländern (aBL) rd. $120 €$ geringer ausfiel als bei den Bestandsrenten desselben Jahres, erhielten die Zugangsrentner in den neuen Bundesländern (nBL) einen um rd. $200 €$ geringeren Zahlbetrag als die entsprechenden Bestandsrentner. Eine höhere Arbeitslosigkeit sowie geringere Entgelte in den nBL wirken sich unmittelbar auf die Rente aus und zeigen, dass die Rente Spiegelbild des Erwerbslebens ist. Bei den Frauen - sowohl in den alten Bundesländern wie auch in den neuen - ist die Lage von Bestands- und Zugangsrentnerinnen gleichermaßen desolat. Beachtlich ist, dass sich eine Erhöhung der Frauenerwerbsquote in den aBL auf die Rentenzahlbeträge nicht auswirkt. Frauen arbeiten mehr, erhalten aber keine höheren Renten. Maßgeblicher Grund hierfür sind die immer noch gerin geren Erwerbseinkommen von Frauen sowie die von ihnen verstärkt ausgeübten prekären Beschäftigungsverhältnisse und Teilzeitarbeitsverhältnisse. Dies wird insbesondere beim sogenannten Minijob deutlich: Trotz der ab 1.1.2013 ein-

\footnotetext{
1 Vgl. AltersvermögensGesetz (AVmG) v. 26.6.2001, BGBI. I, S. 1310, dessen wesentlicher Inhalt die Einführung der Riester-Rente, Normierung eines Rechtsanspruchs auf Entgeltumwandlung und die Einführung einer bedarfsorientierten Grundsicherung war, sowie das AltersvermögensergänzungsGesetz (AVmEG) v. 21.3.2001, BGBI. I, S. 403, das die Neugestaltung der Rentenformel, das Rentensplitting unter Ehegatten und die Neuregelungen zum Hinterbliebenenrentenrecht zum Gegenstand hatte. Die Trennung in ein zustimmungsfreies und ein zustimmungspflichtiges Gesetz erfolgte aufgrund der unterschiedlichen Mehrheitsverhältnisse in Bundestag und Bundesrat.
}

2 Der Rentenzahlbetrag ist die Bruttorente abzüglich des von den Rentenbeziehenden zu tragenden Beitrags zur Kranken- und Pflegeversicherung (rd. $10 \%$ ) 
geführten Versicherungspflicht führt ein Minijob zu keiner existenzsichernden sozialen Absicherung. Um mit einem versicherungspflichtigen 300-€-Minijob die Grundsicherungsgrenze von rd. $690 €$ netto zu erreichen, müsste dieser Minijob 250 Jahre, ein 450-€-Minijob 170 Jahre ausgeübt werden.

\section{Die politische Debatte}

Die sinkenden Rentenzahlbeträge wurden von der Politik lange ignoriert: von der Regierungskoalition, um ihre eigene Untätigkeit bei der Problemlösung zu verschleiern, von der oppositionellen SPD und Bündnis 90/Die Grünen zunächst, um einer Diskussion über die Folgen ihres eigenen Handelns in der Legislaturperiode 1998-2002 auszuweichen. Die zuständige CDU-Sozialministerin wiederum hat im Jahr 2012 mit der Zuschussrente/Lebensleistungsrente ein Instrument vorgestellt, das nach einhelliger Meinung aller sozialpolitscher Akteurinnen und Akteure nicht geeignet ist, Altersarmut zu bekämpfen: Die (erwerbsbiografischen) Voraussetzungen, um die Zuschussrente überhaupt beziehen zu können, sind so hoch, dass diejenigen, die die finanzielle Unterstützung bräuchten, sie in der Regel nicht erfüllen können. Und diejenigen, die die Voraussetzungen erfüllen, beziehen in der Regel Alterseinkünfte oberhalb der festgesetzten Grenze von $850 €$. Gleichzeitig wurde die von der Frauenunion der CDU und der CSU geforderte sogenannte Mütterrente ${ }^{3}$ nicht mehr rechtzeitig in das Gesetzgebungsverfahren eingebracht.

Bei der SPD, in deren Regierungszeit mit Bündnis 90/Die Grünen die Normierung der „Beitragssatzstabilität“ fällt, wurde ein innerparteilicher Diskurs darüber geführt, die Regierungspolitik von damals zu korrigieren. Mit ihren Vorschlägen im Regierungsprogramm ${ }^{4}$ will die SPD in einem ersten Schritt u.a. die Rente nach Mindesteinkommen verlängern, die rentenrechtliche Anerkennung von Zeiten des Arbeitslosengeld (ALG)-II-Bezugs verbessern, das geltende Rentenniveau bis 2020 festschreiben und die Rente mit 67 für lange Zeit aussetzen.

\section{Erwerbsminderung führt oftmals direkt in die Armut}

Nicht nur die Altersrenten zeigen Reformbedarf, sondern auch die Erwerbsminderungsrenten (EM-Renten). Letztere sollen das Einkommen (ganz oder teilweise) ersetzen, wenn die Erwerbsfähigkeit nicht mehr in erforderlichem Maße vorhanden ist. Jedoch: Der bundesdurchschnitt- liche Zahlbetrag voller Erwerbsminderungsrenten lag im Zugang 2011 für Männer und Frauen bei $634 €$, wobei eine derart große Spannbreite wie bei den Altersrenten nicht zu verzeichnen ist. ${ }^{5}$ Entscheidend hierbei ist, dass die Grundsicherungsgrenze von knapp $690 €$ netto deutlich unterschritten wird. Von den knapp 800.000 Grundsicherungsempfängerinnen und -empfängern im Jahr 2010 bezog knapp die Hälfte Grundsicherung wegen Erwerbsminderung. ${ }^{6}$ Obwohl die Anhebung der Zahlbeträge der EM-Renten von allen maßgeblichen Parteien gefordert wurde, hat es die Bundesregierung über die gesamte Legislaturperiode versäumt, dies gesetzgeberisch zu regeln.

\section{Diskussion um die „Rente mit 67“}

In der öffentlichen Diskussion spielt die Anhebung des Renteneintrittsalters, die bereits für den Geburtsjahrgang 1947 im Jahr 2012 begann und im Jahr 2029 abgeschlossen sein soll, eine wichtige Rolle. ${ }^{7}$ Die Anhebung der Regelaltersrente vom vollendeten 65 . auf das vollendete 67 . Lebensjahr und parallel der weiteren Altersrenten wird von den Betroffenen - zu Recht - als Rentenkürzung empfunden. Es wurde versäumt, vor Anhebung der Altersgrenzen auf dem Arbeitsmarkt und in den Betrieben die grundlegenden Voraussetzungen dafür zu schaffen, dass Ältere überhaupt länger sozialversicherungspflichtig erwerbstätig sein können. Dass es noch ein weiter Weg bis zu einer zufriedenstellenden Beschäftigung Älterer ist, wird daran deutlich, dass nach Daten der Bundesanstalt für Arbeit und des Statistischen Bundesamtes aktuell nur $27 \%$ der 60-Jährigen in Vollzeitarbeit beschäftigt sind. Die Vollzeit-Beschäftigungsquoten nehmen mit dem Lebensalter kontinuierlich ab und liegen bei den 64-Jährigen nur noch bei 9,9\%.

Von einzelnen Akteurinnen und Akteuren werden anstelle des Aussetzens bzw. der Rücknahme der „Rente mit 67“ Möglichkeiten eines flexiblen und vorzeitigen Ausstiegs, z.B. durch die „abschlagsfreie Rente mit 63 “ nach 45 Versicherungsjahren oder die Kombination von Teilrente und Erwerbsarbeit, diskutiert. Von diesen Vorschlägen würden aber nur Versicherte profitieren, die eine längere Erwerbsbiografie vorweisen können bzw. deren Rentenhöhe eine durch Abschläge geminderte Teilrente erlaubt. In beiden Fällen wären Frauen benachteiligt, denn sie weisen durchschnittlich kürzere Erwerbszeiten auf und haben einen geringeren finanziellen Spielraum. Wer also ein Beibehalten der „Rente mit 67 “ und stattdessen Modelle des flexiblen Über- gangs propagiert, diskriminiert Frauen. Erforderlich ist beides: ein Aussetzen bzw. Abschaffen

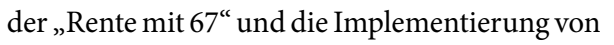
Modellen des flexiblen Übergangs.

\section{Rentenanpassung zum 1.7.2013}

In den alten Bundesländern wurden die Renten zum 1.7.2013 um nur 0,25 \%, in den neuen Ländern um 3,29\% angehoben. Eine Rente von $1.000 €$ steigt damit in den aBL um 2,50€, in den nBL um 32,90 $€$ an. ${ }^{8}$ Die Rentenanpassung in den aBL gleicht also den Kaufkraftverlust bei Weitem nicht aus.

Auf den ersten Blick erscheint dies als eine Benachteiligung der Rentnerinnen und Rentner in den aBL. Die unterschiedlichen Anpassungssätze sind vor allem darauf zurückzuführen, dass die beitragspflichtigen Löhne und Gehälter in den nBL deutlich stärker als in den aBL angestiegen sind. Das hat u. a. folgende Gründe:

(1) Zum 1.1.2011 wurde die Beitragsbemessungsgrenze (BBG) Ost um $1.800 €$ angehoben, die BBG West nicht. Entsprechend stiegen auch die beitragspflichtigen Entgelte in den nBL.

(2) Da in den beitragspflichtigen Entgelten auch die Entgelte aus Minijobs berücksichtigt werden und Minijobs in den aBL weit deutlicher ansteigen als in den $\mathrm{nBL}$, wirkt sich auch dies negativ auf die Anpassung in den aBL aus.

3 Mit der Mütterrente wird die Verbesserung der rentenrechtlichen Bewertung bis hin zur Gleichbehandlung von Kindererziehungszeiten für vor 1992 geborene Kinder (1 Entgeltpunkt-EP) mit denen nach 1991 Geborenen (3 EP) gefordert.

4 Das Regierungsprogramm der SPD, beschlossen auf dem außerordentlichen Parteitag am 14.4.2013 in Augsburg, erfüllt weitgehend die vielfältigen gewerkschaftlichen Forderungen.

5 Vgl. Deutsche Rentenversicherung Bund (2012) (Hrsg.): Rentenversicherung in Zeitreihen: DRVSchriften Band 22, Oktober 2012, S. 128.

6 Vgl. Statistisches Bundesamt (2011).

7 RV-Altersgrenzenanpassungsgesetz v. 20.4.2007 BGBI. I, S. 544.

8 Die aktuellen Rentenwerte (aRW) betragen damit ab 1.7.2013 in den aBL: $28,14 €$ (bis 30.6.2013: $28,07 €$ ) und in den $\mathrm{nBL} 25,74 €$ (bis 30.6.2013: $24,92 €)$. Der aRW Ost beträgt damit 91,5 \% des aRW West (bis 30.6.13: 88,8\%). 
Dies zeigt, dass die höchst unterschiedliche Anpassung auch aus einer Politik der Ausweitung der prekären Beschäftigungsverhältnisse und den im Referenzzeitraum immer noch relativ geringen Lohn- und Gehaltssteigerungen resultiert. Rund 23 Jahre nach der Deutschen Einheit ist ein bundeseinheitliches Rentenrecht nicht nur aus staatsrechtlichen Gründen, sondern auch aus Akzeptanz- und Gerechtigkeitserwägungen dringend erforderlich.

\section{Fazit}

Neben den hier behandelten Stichworten und Problemen umfasst die aktuelle Alterssicherungsdebatte noch weitaus mehr Themen, wie z.B. die Anrechnung jeglichen Einkommens auf die Grundsicherung und damit die mangelnde Attraktivität von zusätzlicher Vorsorge, aber auch die Aktivitäten auf europäischer Ebene. ${ }^{9}$ Doch bereits anhand dieser Ausführungen wird deutlich: Eine der vordringlichsten Aufgaben der Politik ist es, eine Ordnung auf dem Arbeitsmarkt herzustellen, die die Integration Älterer in das Beschäftigungssystem ermöglicht, sowie ein Rentenniveau festzulegen, mit dem ein würdiges und angemessenes Leben im Alter geführt werden kann. Eine kürzungsfaktorenfreie Rentenanpassungsformel ist dazu ebenso notwendig wie das Bewusstsein, dass alle Maßnahmen, die Leis-

9 Späterer Renteneintritt und Stärkung der kapitalgedeckten Altersvorsorge sind Maßnahmen, die sowohl von nationalen Politikern wie auch von der EU-Kommission und der OECD vorgeschlagen werden, um die Alterssicherungssysteme zukunftsfest zu machen. tungsverbesserungen zum Ziel haben, Geld kosten, das je nach Risikozuordnung aus Steuern oder Beiträgen aufgebracht werden muss. Weder die Vermeidung noch die Bekämpfung von $\mathrm{Al}$ tersarmut gibt es zum Nulltarif. Die Menschen wollen echte Reformen und eine realistische Aussicht auf eine gute Rente.

\section{AUTORIN}

JUDITH KERSCHBAUMER, Dr. jur., ist Leiterin des Bereichs Sozialpolitik in der Bundesverwaltung der Vereinten Dienstleistungsgewerkschaft (ver.di), Rechtsanwältin und Dozentin. Arbeitsschwerpunkte: Alterssicherung von Frauen, Rentenrecht und Deutsche Einheit, betriebliche Altersversorgung.

@judith.kerschbaumer@verdi.de 